\title{
Low DHEAS levels are associated with depressive symptoms in elderly Chinese men: results from a large study
}

\author{
Samuel YS Wong ${ }^{1}$, Jason C Leung ${ }^{2}$, Timothy Kwok ${ }^{3}$, Claes Ohlsson ${ }^{4}$, Liesbeth Vandenput ${ }^{4}$, Ping C Leung ${ }^{2}$ \\ and Jean Woo ${ }^{5}$
}

This study investigated the association between depressive symptoms in elderly Chinese men and the total testosterone, dehydroepiandrosterone (DHEA), DHEA sulphate (DHEAS), oestradiol and sex hormone-binding globulin (SHBG) levels, and the free androgen index. Cross-sectional data from 1147 community-dwelling elderly men, aged 65 and older, were used. Depressive symptoms were measured using the Chinese Geriatric Depression Scale (GDS). Total testosterone, free testosterone, DHEA, DHEAS, total oestradiol, the free androgen index and SHBG levels were assessed. DHEA was significantly associated with GDS score, and there was a trend towards DHEAS association, but this was not significant $(\beta=-0.110, P=0.015 ; \beta=-0.074, P=0.055)$. However, no association was seen between depressive symptoms and total testosterone levels, free testosterone levels, oestradiol levels or SHBG levels. In terms of the presence of clinically relevant depressive symptoms, there were no statistically significant differences between patients in the lowest quartile of sex steroid hormone levels and those in other quartiles of sex steroid hormone levels. Similarly to Western studies, our study shows that DHEA and DHEAS levels are associated with depressive symptoms. Asian Journal of Andrology (2011) 13, 898-902; doi:10.1038/aja.2011.116; published online 29 August 2011

Keywords: Chinese; dehydroepiandrosterone; depression; elderly men; testosterone

\section{INTRODUCTION}

Depression is a prevalent disorder in the elderly and causes significant morbidity and mortality. ${ }^{1}$ There is epidemiological evidence of an association between depression or clinically relevant depressive symptoms and low levels of free testosterone in elderly men. ${ }^{2}$ There are theoretical grounds to support the hypothesis that low testosterone levels can lead to depression. First, oestrogens and androgens affect mood and behaviour, and studies have demonstrated relationships between the levels of sex steroid hormones and neurotransmitters, including serotonin, both in humans and other animals. ${ }^{3}$ Second, the well-established gender differences in the prevalence of depression suggest that testosterone may play a protective role in the pathogenesis of depression in men, resulting in a lower prevalence of depression in men than in women, although other social factors, such as differences in socialisation and help-seeking behaviours between the two sexes, may also play important roles. ${ }^{4}$ Furthermore, the high prevalence of depression in elderly men, ${ }^{5}$ which coincides with a physiological drop in testosterone levels, suggests that there may be a relationship between low testosterone levels and depression in men, although more research is needed to confirm whether this relationship exists. ${ }^{6}$

Several large studies have explored the relationship between depression and low levels of testosterone. The Rancho Bernardo study ${ }^{7}$ demonstrated the association between bioavailable testosterone and depressive symptoms using a cross-sectional design. Morsink et al. ${ }^{8}$ showed that dehydroepiandrosterone sulphate (DHEAS) levels are inversely associated with depressive symptoms in both elderly men and women. Moreover, men in the lowest testosterone quartile reported significantly more depressive symptoms than men in the other total testosterone quartiles. In the Health in Men study, Almeida et al. ${ }^{9}$ showed that low free testosterone concentrations are associated with depressive symptoms in older men.

In addition to cross-sectional studies, Shores et al. ${ }^{10}$ demonstrated an association between baseline testosterone levels and incident depression in elderly Americans. Recently, using data from the Longitudinal Aging Study in Amsterdam, Joshi et al. ${ }^{4}$ demonstrated an association between testosterone levels and depressive symptoms.

However, despite the findings from these studies, not all studies have shown statistically significant results. ${ }^{11,12}$ Moreover, to our knowledge, no large epidemiological studies have been conducted outside the United States or Europe or in Asians. The present study investigated the association between sex steroid hormone levels and depressive symptoms cross-sectionally in a cohort of elderly Chinese men. We hypothesized that low testosterone levels are associated with depressive symptoms in elderly Chinese men.

${ }^{1}$ School of Public Health and Primary Care, School of Public Health, The Chinese University of Hong Kong, Hong Kong, China; ${ }^{2}$ ockey Club Centre for Osteoporosis Care and Control, The Chinese University of Hong Kong, Hong Kong, China; ${ }^{3}$ Department of Medicine and Therapeutics, The Chinese University of Hong Kong, Hong Kong, China; ${ }^{4}$ Center for Bone Research, Department of Internal Medicine, University of Gothenburg, Gothenburg SE-413 45, Sweden and ${ }^{5}$ Division of Geriatrics, Department of Medicine and Therapeutics, The Chinese University of Hong Kong, Hong Kong, China

Correspondence: Dr SYS Wong (yeungshanwong@cuhk.edu.hk)

Received: 23 February 2011; Revised: 11 May 2011; Accepted: 29 June 2011; Published online: 29 August 2011 


\section{MATERIALS AND METHODS}

\section{Subjects}

The methodology of this study has been described previously. ${ }^{13} \mathrm{Mr}$ Os (Osteoporosis in Men) is a large prospective study conducted in Hong Kong, China, initially to investigate risk factors that are associated with osteoporosis and fractures in elderly Chinese. Since its inception, the study has been expanded to cover other areas that are of interest concerning the health of the elderly. In brief, 2000 Chinese men who were 65 years and older were recruited. Subjects were interviewed between August 2001 and February 2003 at baseline using a standardized, structured questionnaire. Informed consent was obtained from all subjects. Participants were randomly selected to have their blood taken at a baseline visit to measure total serum testosterone, free testosterone, DHEAS, DHEA, oestradiol and sex hormone-binding globulin (SHBG) levels. This study was approved by the Ethics Committee of the Faculty of Medicine at the Chinese University of Hong Kong.

Participants were excluded from our analyses if no sex hormone data could be collected $(n=511)$, if they were cognitively impaired $(n=159)$ or if they had received testosterone injections $(n=2)$, androgen antagonists $(n=4)$ oral corticosteroids $(n=7)$ or any medication for the prostate $(n=170)$. Complete data were available for $1147 \mathrm{sub}$ jects in the current study.

\section{Assessments}

The stored sera from 1147 subjects were analysed for precursors (DHEA, DHEAS, androst-5-ene-3 $\beta, 17 \beta$-diol and androstenedione) and bioactive hormones (testosterone, dihydrotestosterone, oestrone and oestradiol). The free androgen index was calculated using the following formula: free androgen index $=100 \times$ (total testosterone/ SHBG) $\times 3.467 \mathrm{nmol}^{-1}$. All hormone assays were performed using mass spectrometry; DHEAS was measured using liquid chromatography-mass spectrometry, and the other hormones were measured using gas chromatography-mass spectroscopy. ${ }^{14,15}$ Standard samples of several concentrations were included in all assay runs to ensure precision and accuracy. Moreover, blinded aliquots of a single serum pool were included in all assay runs and showed no longitudinal trend in any measurement. The coefficients of variation ranged from $3.0 \%$ to $7.4 \%$. Free fractions of testosterone and oestradiol were calculated as described by Södergård et al. ${ }^{16}$

Sociodemographic information, including age, address, place of origin, education level and occupation, was also obtained. Data on weight, height, bone mineral density and body composition were collected. The Chinese Mini-Mental Status Examination ${ }^{17}$ was used as an evaluation of cognitive impairment. An individual with a MiniMental Status Examination score of 23 or less was identified as being demented. Clinically relevant depressive symptoms were diagnosed by face-to-face interviews, using a validated 15-item Chinese version of the Geriatric Depression Scale (GDS), and were defined as being 8 or higher. ${ }^{18}$ Lifestyle characteristics, including smoking status and smoking amount (categorized as former smoker, having smoked at least 100 cigarettes smoked in a lifetime; current smoker; and never having smoked), alcohol consumption (daily frequency of intake of alcohol and other beverages in portion sizes) and physical activity, were also collected. Information on subjects' medical histories was obtained based on self-reports. In addition, details of current medications were collected. Participants were asked to bring all medications, including over-the-counter drugs and Chinese medicines, which were taken regularly in the past 2 weeks to the centre for verification. No participant reported the use of DHEA.

\section{Statistical analysis}

Participant characteristics among the 1147 males were tabulated. To normalize the distributions, sex hormones levels were log-transformed. To normalize the GDS variable, a square-root transformation was performed, although in tables and figures, the actual GDS scores are reported. To investigate the association between depressive symptoms at baseline and the total testosterone, DHEA, DHEAS, DHEA/ DHEAS ratio, oestradiol, free testosterone and SHBG levels and the free androgen index at baseline, linear regression analyses were conducted with and without adjustments for age, education, body mass index (BMI), smoking and chronic diseases that may have confounded this relationship, including cardiovascular disease, diabetes and a history of prostate cancer. Multiple regression analysis was conducted on the associations between the levels of sex hormones and depressive symptoms, adjusting for age, education, BMI, smoking status and chronic diseases. To examine whether associations were linear or whether any 'threshold effects' appeared, analysis of covariance was used to examine the square-root GDS scores for those in the lowest quartile against those in other quartiles of sex steroid hormone levels. ${ }^{8}$ Statistical analysis was carried out using the statistical package SAS, version 9.1.3 and $P$ value under 0.05 was considered as significant.

\section{RESULTS}

The basic sociodemographic information of the sample of 1147 elderly men for which sex steroid hormone data are available is shown in Table 1. The median (interguartile range) of the total testosterone level in this sample was $5.18 \mathrm{ng} \mathrm{ml}^{-1}\left(2.44 \mathrm{ng} \mathrm{ml}^{-1}\right)$. About $8.0 \%$ of this sample had clinically relevant depressive symptoms.

Table 2 shows the results from the linear regression analyses that assessed the association between the levels of sex steroid hormones and depressive symptoms before and after adjustments for age, education,

\section{Table 1 Baseline characteristics $(n=1147)$}

\begin{tabular}{|c|c|}
\hline Variable & Value \\
\hline Age (year) ${ }^{\mathrm{a}}$ & $71.99 \pm 4.71$ \\
\hline $\mathrm{BMI}^{\mathrm{a}}$ & $23.39 \pm 3.16$ \\
\hline Education primary or below ${ }^{\mathrm{b}}$ & $661(57.63)$ \\
\hline \multicolumn{2}{|l|}{ Smoking status ${ }^{\mathrm{b}}$} \\
\hline Never smoker & 415 (36.18) \\
\hline Past smoker & $591(51.53)$ \\
\hline Current smoker & $141(12.29)$ \\
\hline Prostate cancer in the past ${ }^{\mathrm{b}}$ & $13(1.13)$ \\
\hline $\mathrm{Ml} /$ angina/CHF ${ }^{\mathrm{b}}$ & $192(16.74)$ \\
\hline Diabetes $^{b}$ & $170(14.82)$ \\
\hline At risk for depression, GDS $\geqslant 8^{b}$ & $92(8.02)$ \\
\hline GDS score ${ }^{c}$ & $2(3)$ \\
\hline Total testosterone $\left(\mathrm{ng} \mathrm{ml}^{-1}\right)^{\mathrm{c}}$ & $5.18(2.44)$ \\
\hline SHBG $\left.(\mathrm{nmol} \mathrm{I})^{-1}\right)^{c}$ & $47.81(23.34)$ \\
\hline Free Androgen Index ${ }^{c}$ & $39.05(15.10)$ \\
\hline DHEA $\left(n g \mathrm{ml}^{-1}\right)^{\mathrm{c}}$ & $1.76(1.20)$ \\
\hline DHEAS $\left(m g \mathrm{ml}^{-1}\right)^{\mathrm{c}}$ & $0.91(0.68)$ \\
\hline DHEA/DHEAS ratio ${ }^{c}$ & $2.01(1.10)$ \\
\hline Oestradiol $\left(p g \mathrm{ml}^{-1}\right)^{\mathrm{c}}$ & $23.02(9.60)$ \\
\hline Free testosterone $\left(\mathrm{ng} \mathrm{dl^{-1 } ) ^ { c }}\right.$ & $10.63(4.01)$ \\
\hline Free oestradiol $\left(\mathrm{pg} \mathrm{ml^{-1 }}\right)^{\mathrm{c}}$ & $0.54(0.20)$ \\
\hline
\end{tabular}

Abbreviations: BMI, body mass index; CHF, congestive heart failure; DHEA, dehydroepiandrosterone; DHEAS, dehydroepiandrosterone sulphate; GDS, Geriatric Depression Scale; MI, myocardial infarction; SHBG, sex hormone-binding globulin. ${ }^{a}$ Values are presented as mean \pm s.d.

${ }^{b}$ Values are presented as $n(\%)$

${ }^{\mathrm{C}}$ Values are presented as media (interquartile range). 
Table 2 Linear regression results of the association between the levels of sex hormones ${ }^{\mathrm{a}}$ and the depressive symptoms (GDS) ${ }^{\mathrm{b}}$

\begin{tabular}{|c|c|c|c|c|}
\hline & \multicolumn{2}{|c|}{ Unadjusted results } & \multicolumn{2}{|c|}{ Adjusted ${ }^{c}$ results } \\
\hline Total testosterone $\left(\mathrm{ng} \mathrm{ml}^{-1}\right.$ ) & $0.020(-0.093,0.133)$ & 0.730 & $-0.032(-0.156,0.091)$ & 0.607 \\
\hline Free androgen index & $0.027(-0.099,0.153)$ & 0.677 & $0.018(-0.116,0.152)$ & 0.788 \\
\hline $\operatorname{DHEA}\left(\mathrm{ng} \mathrm{ml^{-1 }}\right)$ & $-0.134(-0.221,-0.047)$ & 0.003 & $-0.110(-0.199,-0.022)$ & 0.015 \\
\hline DHEAS ( $\mathrm{mg} \mathrm{m}^{-1}$ ) & $-0.090(-0.165,-0.015)$ & 0.018 & $-0.074(-0.149,0.001)$ & 0.055 \\
\hline Free testosterone $\left(\mathrm{ng} \mathrm{dl}^{-1}\right)$ & $0.020(-0.106,0.146)$ & 0.758 & $-0.021(-0.155,0.114)$ & 0.761 \\
\hline Free oestradiol $\left(\mathrm{pg} \mathrm{ml}^{-1}\right)$ & $0.092(-0.040,0.225)$ & 0.173 & $0.081(-0.055,0.216)$ & 0.244 \\
\hline
\end{tabular}

Abbreviations: BMI, body mass index; CHF, congestive heart failure; DHEA, dehydroepiandrosterone; DHEAS, dehydroepiandrosterone sulphate; GDS, Geriatric Depression Scale; MI, myocardial infarction; SHBG, sex hormone-binding globulin.

${ }^{\text {a }}$ Sex hormone levels were log transformed.

${ }^{\mathrm{b}}$ The square root of the GDS was used.

${ }^{\mathrm{c}}$ Adjusted for age, education, BMI, smoking, MI/angina/CHF, diabetes and previous diagnosis of prostate cancer.

BMI, smoking and chronic diseases. There was a significant inverse association between DHEA level and GDS score (unadjusted $\beta=-0.134, P=0.003)$, which was attenuated after adjustment for the factors noted above $(\beta=-0.110, P=0.015)$. Similarly, there was a significant inverse association between DHEAS level and GDS score (unadjusted $\beta=-0.090, P=0.018$ ), which was also attenuated after adjustment $(\beta=-0.074, P=0.055)$. However, total testosterone, free testosterone, SHBG and oestradiol levels and the free androgen index were not significantly associated with GDS scores.

Similar to the method used by Morsink et al., ${ }^{8}$ we divided the sex steroid hormone levels into quartiles to examine whether there was a 'threshold effect' or a 'gradient effect' in the association between sex steroid hormone levels and depressive symptoms. Depressive symptom scores among patients in the lowest quartile of any sex steroid hormone levels collected in this study were not significantly different from those for patients in higher quartiles of sex steroid hormone levels or the free androgen index before or after adjusting for potential confounding variables $(P>0.05$; Table 3$)$. Figure 1 shows the relationships between various sex hormone levels and GDS scores. There were no significant threshold effects in the association between sex steroid hormone levels and depressive symptoms.

\section{DISCUSSION}

This study explored the association between depressive symptoms and the total testosterone, free testosterone, DHEA, DHEAS, oestradiol, free testosterone and SHBG levels and the free androgen index in a sample of community-dwelling elderly Chinese men. To our knowledge, we are the first to explore these relationships in Asians using data from a large prospective cohort. In contrast to previous findings from studies of large cohorts, ${ }^{4,8}$ we found a significant inverse association only between depressive symptoms (defined by GDS score) and DHEA or DHEAS, and we did not find an association between depressive symptoms and the serum levels of total testosterone, oestradiol, free testosterone or the free androgen index in these Chinese elderly men.

Our finding of an inverse association between DHEA or DHEAS level and depressive symptoms confirms the relationship that has previously been shown in Caucasians and African-Americans. ${ }^{8,19,20}$ We measured both DHEA and DHEAS levels, because DHEA is the precursor of DHEAS. DHEAS is the sulphated version of DHEA with a conversion that is reversibly catalysed by sulphotransferase (SULT2A1). Indeed, it has been suggested that among all of the sex steroid hormones that are associated with depressive symptoms in the elderly, findings on the association between DHEAS/DHEA levels and depressive symptoms are the most consistent. ${ }^{19}$

DHEA has antidepressant effects through the modulation of neurotransmitter systems in the brain in animal studies. ${ }^{21}$ Overall, DHEAS increases the excitability of the brain and has effects similar to antidepressants on the neurotransmitter system, resulting in an improvement in mood. Other possibilities for the association between low DHEAS level and depressive symptoms include the possibility that depressive symptoms could cause lower DHEAS levels or the possibility that the relationship is confounded by the presence of medical comorbidity or a lack of physical activity or malnutrition, which is common in the elderly and could have affected both the DHEAS levels and the depressive symptoms. Previous research has also shown that the presence of multiple chronic conditions lowers DHEAS levels and increases depressive symptoms. ${ }^{22}$

In further support of the idea that DHEA/DHEAS level has a role in depression, a recent randomized controlled trial ${ }^{23}$ in the United Kingdom showed that DHEA improved subjective mood and decreased the evening cortisol concentration, which is elevated in people with depression. ${ }^{24}$

The reason for the lack of an association between the free androgen index and depressive symptoms is not known, but this result could result from the indirect measurement of the free androgen index in our study. We calculated the free androgen index using a formula ${ }^{16}$ and did not directly measure free testosterone or bioavailable testosterone. Moreover, no measurement of albumin was taken in our study, and this measurement is needed to calculate free testosterone using the method of Vermeulen et al., ${ }^{25}$ which was also used in a recent study by Joshi et al. ${ }^{4}$

We do not know why our study did not find an association between total testosterone levels and depressive symptoms. The value of the mean level of total testosterone in our study was comparable to or higher than the levels of total testosterone from previous studies (e.g., mean total testosterone $=5.47 \mathrm{ng} \mathrm{l}^{-1}$ or $18.96 \mathrm{mmol}^{-1}$ in our study, $3.82 \mathrm{ng} \mathrm{l}^{-1}$ in Morsink et al.'s study and $14.61 \mathrm{mmoll}^{-1}$ in Joshi et al.'s study) that demonstrated this association. ${ }^{4,8}$ The presence of very few hypogonadal men in our study may explain the lack of association. However, the relationship between total testosterone level and depressive symptoms is inconsistent in the literature, with some studies reporting a significant association ${ }^{8}$ and others reporting no association. $^{7,26,27}$ 
Table 3 Depressive symptom scores for men in the lowest quartile of sex hormone levels versus those in other quartiles ${ }^{\mathrm{a}}$

\begin{tabular}{|c|c|c|c|c|c|c|c|}
\hline & \multirow{3}{*}{$\mathrm{n}$} & \multicolumn{6}{|c|}{ Depressive symptoms ${ }^{b}$} \\
\hline & & \multicolumn{3}{|c|}{ Unadjusted } & \multicolumn{3}{|c|}{ Adjusted $^{c}$} \\
\hline & & Mean & $\beta(95 \% C l)$ & $P$ & Mean & $\beta(95 \% C l)$ & $P$ \\
\hline \multicolumn{8}{|c|}{ Total testosterone $\left(\mathrm{ng} \mathrm{ml}^{-1}\right.$ ) } \\
\hline Lowest quartile & 284 & 2.97 & Ref. & & 3.06 & Ref. & \\
\hline Other quartiles & 863 & 2.78 & $-0.058(-0.177,0.062)$ & 0.342 & 2.75 & $-0.083(-0.208,0.041)$ & 0.189 \\
\hline \multicolumn{8}{|l|}{ SHBG $\left(n m o l I^{-1}\right)^{a}$} \\
\hline \multicolumn{8}{|c|}{ Free Androgen Index ${ }^{a}$} \\
\hline Lowest quartile & 286 & 2.91 & Ref. & & 2.87 & Ref. & \\
\hline Other quartiles & 860 & 2.80 & $-0.024(-0.143,0.095)$ & 0.689 & 2.81 & $-0.007(-0.129,0.115)$ & 0.907 \\
\hline \multicolumn{8}{|l|}{ DHEA $\left(n g \mathrm{ml}^{-1}\right)$} \\
\hline Lowest quartile & 281 & 2.94 & Ref. & & 2.92 & Ref. & \\
\hline Other quartiles & 866 & 2.79 & $-0.075(-0.194,0.045)$ & 0.221 & 2.79 & $-0.061(-0.181,0.059)$ & 0.320 \\
\hline Lowest quartile & 286 & 2.81 & Ref. & & 2.79 & Ref. & \\
\hline Other quartiles & 861 & 2.83 & $-0.028(-0.147,0.091)$ & 0.645 & 2.83 & $-0.018(-0.136,0.099)$ & 0.758 \\
\hline \multicolumn{8}{|c|}{ Oestradiol (pg ml ${ }^{-1}$ ) } \\
\hline Lowest quartile & 285 & 2.79 & Ref. & & 2.83 & Ref. & \\
\hline Other quartiles & 862 & 2.84 & $0.031(-0.088,0.151)$ & 0.605 & 2.82 & $0.010(-0.108,0.128)$ & 0.869 \\
\hline \multicolumn{8}{|c|}{ Free testosterone $\left(n g d^{-1}\right)^{a}$} \\
\hline Lowest quartile & 284 & 3.00 & Ref. & & 3.04 & Ref. & \\
\hline Other quartiles & 862 & 2.77 & $-0.038(-0.158,0.081)$ & 0.530 & 2.76 & $-0.047(-0.168,0.075)$ & 0.450 \\
\hline \multicolumn{8}{|c|}{ Free oestradiol $\left(\mathrm{pg} \mathrm{ml}^{-1}\right)^{\mathrm{a}}$} \\
\hline Lowest quartile & 269 & 2.73 & Ref. & & 2.70 & Ref. & \\
\hline Other quartiles & 877 & 2.86 & $0.068(-0.054,0.189)$ & 0.274 & 2.86 & $0.076(-0.046,0.197)$ & 0.222 \\
\hline
\end{tabular}

Abbreviations: BMI, body mass index; CHF, congestive heart failure; DHEA, dehydroepiandrosterone; DHEAS, dehydroepiandrosterone sulphate; GDS, Geriatric Depression Scale; MI, myocardial infarction; SHBG, sex hormone-binding globulin; Ref., reference group.

a Sample size for SHBG, free androgen index, free testosterone and free oestradiol is 1146 because of one missing data.

${ }^{\mathrm{b}}$ Analyses based on square root of GDS scores, but actual GDS scores are reported.

${ }^{\mathrm{c}}$ Adjusted for age, education, BMI, smoking, MI/angina/CHF, diabetes and previous diagnosis of prostate cancer.

Our present study has several limitations. First, we defined depression as scoring higher than the standard cutoff score with the use of a validated Chinese screening instrument for detecting depressive symptoms in elderly Chinese; there may have been an underestimation of depression if these Chinese elderly did not present with psychological symptoms or complaints. It should be noted that the prevalence of depression in our sample was lower than the prevalence that has been reported previously in Hong Kong in elderly Chinese, ${ }^{28}$ probably as a result of recruiting physiologically well-functioning elderly men in our study. Second, this was simply a cross-sectional study, and a causal relationship between sex steroid hormone levels and depressive symptoms could not be derived. Third, we did not make direct measurements of free testosterone and albumin levels, which may have affected the accuracy of our calculations of the free or bioavailable testosterone levels. Fourth, we only crudely assessed the presence of chronic comorbidities, and medical comorbidities may have

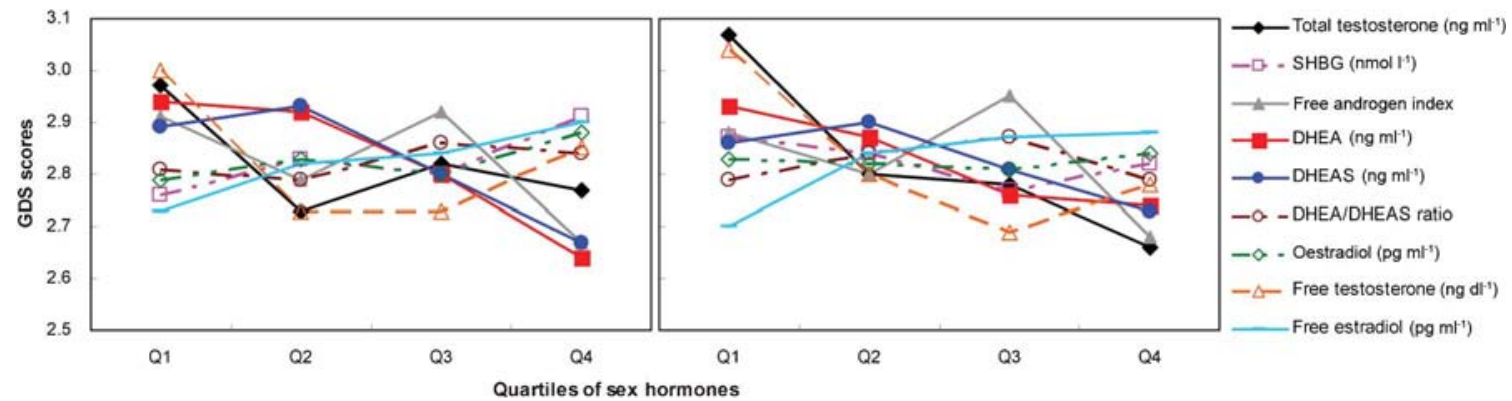

Figure 1 Depression symptom scores by quartiles of sex hormones. (a) Relationship of depression symptom scores and quartiles of sex hormones, $P$ value test for trend for $\mathrm{DHEA}=0.038$. (b) Relationship of depression symptom scores and quartiles of sex hormones, adjusted for age, education, BMI, smoking, MI/angina/CHF, diabetes and previous diagnosis of prostate cancer. BMI, body mass index; CHF, congestive heart failure; DHEA, dehydroepiandrosterone; DHEAS, dehydroepiandrosterone sulphate; MI, myocardial infarction; SHBG, sex hormone-binding globulin. 
affected the levels of sex hormones. Fifth, our sample of men was not recruited by random sampling, and 511 participants were excluded because serum samples were not available for hormone measurement; therefore, it is possible that there is selection bias in this study.

We found a significant association between the DHEA level and depressive symptoms in elderly Chinese. Further longitudinal studies in various ethnic groups are required to further evaluate this relationship.

\section{AUTHOR CONTRIBUTIONS}

All authors contributed equally to this work. SYSW, TK, PCL, and JW designed and performed experiments; SYSW, JCL, TK, CO, and LV analyzed data; SYSW, JCL, TK, and PCL wrote the manuscript.

\section{COMPETING FINANCIAL INTERESTS}

All co-authors have no financial conflicts or other conflicts of interest in this area.

\section{ACKNOWLEDGMENTS}

This work was supported by an NIH grant and a RGC grant. However, the branches of the NIH and the RGC that were responsible for the funding of the Mr Os study did not have any role in the design and implementation of the study; the collection, management, analysis or interpretation of data; or the preparation, review or approval of the manuscript.

1 World Health Organization. The World Health Report 2001-Mental Health: New Understanding, New Hope. Geneva: World Health Organization; 2011.

2 Amiaz R, Seidman SN. Testosterone and depression in men. Curr Opin Endocrinol Diabetes Obes 2008; 15: 278-83.

3 Fink G, Sumner BE, McQueen JK, Wilson H, Rosie R. Sex steroid control of mood, mental state and memory. Clin Exp Pharmacol Physiol 1998; 25: 764-75.

4 Joshi D, van Schoor NM, de Ronde W, Schaap LA, Comijs HC et al. Low free testosterone levels are associated with prevalence and incidence of depressive symptoms in older men. Clin Endocrinol (Oxf) 2010; 72 232-40.

5 Seidman SN, Walsh BT. Testosterone and depression in aging men. Am J of Geriatr Psychiatry 1999; 7: 18-33.

6 Seidman SN, Araujo AB, Roose SP, McKinlay JB. Testosterone level, androgen receptor polymorphism, and depressive symptoms in middle-aged men: the Massachusetts Male Aging Study. Biol Psychiatry 2001; 50: 371-6.

7 Barrett-Connor E, von Muhlen DG, Kritz-Silverstein D. Bioavailable testosterone and depressed mood in older men: the Rancho Bernardo study. J Clin Endocrinol Metab 1999; 84: 573-7.

8 Morsink LF, Vogelzangs N, Nicklas BJ, Beekman AT, Satterfield S et al. Associations between sex steroid hormone levels and depressive symptoms in elderly men and women: results from the Health ABC study. Psychoneuroendocrinology 2007; 32: 874-83.
9 Almeida OP, Yeap BB, Hankey GJ, Jamrozik K, Flicker L. Low free testosterone concentration as a potentially treatable cause of depressive symptoms in older men: health in men study. Arch General Psychiatry 2008; 65: 283-9.

10 Shores MM, Moceri VM, Sloan KL, Matsumoto AM, Kivlahan DR. Low testosterone levels predict incident depressive illness in older men: effects of age and medical comorbidity. J Clin Psychiatry 2005; 66: 7-14.

11 Breuer B, Martucci C, Wallenstein S, Likourezos A, Libow LS et al. Relationship of endogenous levels of sex hormones to cognition and depression in frail, elderly women. Am J Geriatr Psychistry 2002; 10: 311-20.

12 T'Sjoen GG, de Vos S, Goemaere S, van Pottelbergh I, Dierick M et al. Sex steroid level, androgen receptor polymorphism, and depressive symptoms in healthy elderly men. J Am Geratr Soc 2005; 53: 636-42.

13 Wong SY, Hong A, Leung J, Kwok T, Leung PC et al.Lower urinary tract symptoms and depressive symptoms in elderly men. J Affect Disord 2006; 96: 83-8.

14 Labrie F, Bélanger A, Bélanger P, Bérubé R, Martel C et al. Androgen glucuronides, instead of testosterone, as the new markers of androgenic activity in women. J Steroid Biochem Mol Biol 2006; 99: 182-8.

15 Labrie F, Cusan L, Gomez JL, Martel C, Bérubé R et al. Comparable amounts of sex steroids are made outside the gonads in men and women: strong lesson for hormone therapy of prostate and breast cancer. J Steroid Biochem Mol Biol 2009; 113: 52-6.

16 Södergård R, Bäckström T, Shanbhag V, Carstensen H. Calculation of free and bound fractions of testosterone and estradiol- 17 beta to human plasma proteins at body temperature. J Steroid Biochem 1982; 6: 801-10.

17 Katzman R, Zhang MY, Ouang-Ya-Qu, Wang ZY, Liu WT et al. A Chinese version of the Mini-Mental State Examination: impact of illiteracy in a Shanghai dementia survey. J Clin Epidemiol 1988; 41: 971-8.

18 Lee HB, Chiu HF, Kwok WY, Leung CM. Chinese elderly and the GDS short form: a preliminary study. Clin Gerontologist 1993; 14: 37-9.

19 Barrett-Connor E, von Mühlen D, Laughlin GA, Kripke A. Endogenous levels of dehydroepiandrosterone sulfate, but not other sex hormones, are associated with depressed mood in older women: the Rancho Bernado study. J Am Geriatr Soc 1999; 47: 685-91.

20 Berr C, Lafont S, Debuire B, Dartigues JF, Baulieu EE. Relationships of dehydroepiandrosterone sulfate in the elderly with functional, psychological, and mental status, and short term mortality: a French community based study. Proc Nat Acad Sci USA 1996; 93: 13410-5.

21 Majewska MD. Neurosteroids: endogenous bimodal modulators of the GABAA receptor. Mechanism of action and physiological significance. Prog Neurobiol 1992; 38: 379-95.

22 Roose SP, Glassman AH, Seidman SN. Relationship between depression and other medical illnesses. JAMA 2001; 286: 1687-90.

23 Alhaj HA, Massey AE, McAllister-Williams RH. Effects of DHEA administration on episodic memory, cortisol and mood in healthy young men: a double-blind, placebocontrolled study. Psychopharmacology 2006; 188: 541-51.

24 Young EA, Haskett RF, Grunhaus L, Pande A, Weinberg VM et al. Increased evening activation of the hypothalamic-pituitary-adrenal axis in depressed patients. Arch Gen Psychiatry 1994; 51: 701-7.

25 Vermuelen A, Verdonck L, Kaufman JM. A critical evaluation of simple methods for the estimation of free testosterone in serum. J Clin Endocrinol Metab 1999; 84: 3666-72.

26 Laughlin GA, Barrett-Connor E, Bergstrom J. Low testosterone and mortality in older men. The Rancho Bernardo Study. J Clin Endocrin Metab 2008; 93: 63-75.

27 Travison TG, Araujo AB, Kupelian V, O'Donnell AB, McKinlay JB. The relative contributions of aging, health and lifestyle factors to serum testosterone decline in men. The Massachusetts Male Aging Study. J Clin Endocrinol Metab2007; 92: 54955.

28 Woo J, Ho SC, Lau J, Yuen YK, Chiu H et al. The prevalence of depressive symptoms and predisposing factors in an elderly Chinese population.s1994; 89: 8-13. 